\title{
Mixed Finite Elements for Elasticity in the Stress-Displacement Formulation
}

\author{
Douglas N. Arnold and Ragnar Winther
}

\begin{abstract}
We present a family of pairs of finite element spaces for the unaltered Hellinger-Reissner variational principle using polynomial shape functions on a single triangular mesh for stress and displacement. There is a member of the family for each polynomial degree, beginning with degree two for the stress and degree one for the displacement, and each is stable and affords optimal order approximation. The simplest element pair involves 24 local degrees of freedom for the stress and 6 for the displacement. We also construct a lower order element involving 21 stress degrees of freedom and 3 displacement degrees of freedom which is, we believe, likely to be the simplest possible conforming stable element pair with polynomial shape functions. For all these conforming elements the approximate stress not only belongs to $H$ (div), but is also continuous at element vertices, which is more continuity than may be desired. We show that for conforming finite elements with polynomial shape functions, this additional continuity is unavoidable. To overcome this obstruction, we construct as well some non-conforming stable mixed finite elements, which we show converge with optimal order as well. The simplest of these involves only 12 stress and 6 displacement degrees of freedom on each triangle.
\end{abstract}

\section{Introduction}

The Hellinger-Reissner variational principle characterizes the stress field $\sigma$ and the displacement field $u$ engendered in a planar linearly elastic body occupying a region $\Omega$ by a body load $f$ as the unique critical point of the functional $\mathcal{J}$ : $H(\operatorname{div}, \Omega, \mathbb{S}) \times L^{2}\left(\Omega, \mathbb{R}^{2}\right) \rightarrow \mathcal{R}$ defined by

$$
\mathcal{J}(\tau, v)=\int_{\Omega}\left(\frac{1}{2} A \tau: \tau+\operatorname{div} \tau \cdot v-f \cdot v\right) d x .
$$

Here $\mathbb{S}$ denotes the 3 -dimensional vectorspace of $2 \times 2$ symmetric matrices, in which the stress field takes its values. The displacement field takes its values in $\mathbb{R}^{2}$. Finally, the compliance tensor $A$, which may be variable, is a bounded and uniformly symmetric positive definite operator from $\mathbb{S}$ to itself.

2000 Mathematics Subject Classification. Primary: 65N30, Secondary: 74S05.

Key words and phrases. mixed method, finite element, elasticity.

The first author was supported in part by NSF Grant DMS-0107233. The second author was supported in part by the Research Council of Norway under grant 135420/431. 
A mixed finite element determines an approximate stress field $\sigma_{h}$ and an approximate displacement field $u_{h}$ as a critical point of the functional $\mathcal{J}$ restricted to $\Sigma_{h} \times V_{h} \subset H(\operatorname{div}, \Omega, \mathbb{S}) \times L^{2}\left(\Omega, \mathbb{R}^{2}\right)$. As is well-known, the finite element subspaces $\Sigma_{h}$ and $V_{h}$ must be carefully constructed if the discrete solution is to be well-defined and accurate. They must satisfy the stability conditions of the theory of mixed finite elements, cf. $[\mathbf{9}, \mathbf{1 1}]$.

There have been many efforts, dating back four decades, to develop stable mixed finite elements for the stress-displacement formulation of the plane elasticity system, but these have met with little success. Prior to the work described in this note, the only known stable element pairs used composite elements, in which $\Sigma_{h}$ consists of piecewise polynomials with respect to a finer mesh than used for $V_{h}$. See $[\mathbf{5}, \mathbf{1 4}, \mathbf{1 5}, \mathbf{2 3}]$ for the principle examples of such elements. Notice the striking difference with the situation for mixed method discretizations of $H\left(\operatorname{div}, \Omega, \mathbb{R}^{2}\right) \times$ $L^{2}(\Omega, \mathbb{R})$, for which a number of families of stable element pairs are known, such as the Raviart-Thomas elements [18] and the Brezzi-Douglas-Marini elements [10]. The difficulty in extending such elements from the vector-scalar to the tensorvector case lies with the symmetry of the stress tensor. Because of this, many authors have resorted to the use of modified variational principles in which the symmetry of the stress tensor is enforced only weakly $[\mathbf{1}, \mathbf{4}, \mathbf{1 9}, \mathbf{2 0}, \mathbf{2 1}, \mathbf{2 2}]$, or abandoned altogether [6]. Another modification is analyzed in [16].

The authors have recently constructed a family of stable mixed finite element spaces for the unmodified Hellinger-Reissner formulation which use polynomial shape functions with respect to a single arbitrary triangulation for both stress and displacement [7]. There is a member of the family for each polynomial degree $k \geq 1$. The displacement space $V_{h}$ simply consists of all piecewise polynomial vectorfields of degree at most $k$, with no interelement continuity. The stress space $\Sigma_{h}$ consists of all piecewise polynomials of degree $k+1$ together with some of degree $k+2$, subject to more complicated interelement continuity. The method is of optimal order in that the rate of $L^{2}$ approximation is $O\left(h^{k+1}\right)$ for the displacement and $O\left(h^{k+2}\right)$ for the stress, where $h$ is the mesh size. Even for $k=1$ the stress element is fairly complicated, involving 24 degrees of freedom on each triangle. A slightly simpler element, in which the displacement is sought as a piecewise rigid motion (3 degrees of freedom per triangle), and the stress space involves 21 degrees of freedom per triangle, was also shown to be stable. This method is first order in both stress and displacement.

In an effort to develop simpler stable mixed elements, we investigated nonconforming approximations in [8]. Two elements are developed there, both first order with respect to stress and displacement error in $L^{2}$. For the simpler element, the displacement space consists again of piecewise rigid motion and the stress space involves just 12 degrees of freedom per triangle, specifically, the first two moments of the two components of the normal traction on each edge. These methods are nonconforming in that $\Sigma_{h}$ is not a subspace of $H(\operatorname{div}, \Omega, \mathbb{S})$ due to insufficient interelement continuity. Namely, while the normal component of the normal traction, $n^{T} \sigma n$ has the same value on an edge, no matter which triangle the trace is taken from, the tangential component, $t^{T} \sigma_{n}$, is only weakly continuous. 


\section{Conforming elements}

Let $k$ be a positive integer. For a single triangle $T$ we define spaces of shape functions

$$
\begin{aligned}
\Sigma_{T} & =\mathcal{P}_{k+1}(T, \mathbb{S})+\left\{\tau \in \mathcal{P}_{k+2}(T, \mathbb{S}) \mid \operatorname{div} \tau=0\right\} \\
& =\left\{\tau \in \mathcal{P}_{k+2}(T, \mathbb{S}) \mid \operatorname{div} \tau \in \mathcal{P}_{k}\left(T, \mathbb{R}^{2}\right)\right\}, \quad V_{T}=\mathcal{P}_{k}\left(T, \mathbb{R}^{2}\right),
\end{aligned}
$$

where $\mathcal{P}_{k}(X, Y)$ denotes the space of polynomial functions on $X$ of degree at most $k$ taking values in $Y$.

Clearly $\operatorname{dim} V_{T}=(k+1)(k+2)$. In $[7]$ it is shown that $\operatorname{dim} \Sigma_{T}=\left(3 k^{2}+17 k+\right.$ 28)/2 and that a unisolvent set of local degrees of freedom is given by

- the values of three components of $\tau(x)$ at each vertex $x$ of $T$ (9 degrees of freedom)

- the values of the moments of degree at most $k$ of the two normal components of $\tau$ on each edge $e$ of $T$ (6k+6 degrees of freedom)

- the value of the moments $\int_{T} \tau: \phi d x, \phi \in N_{k}(T)\left(\left(3 k^{2}+5 k-2\right) / 2\right.$ degrees of freedom)

Here

$$
N_{k}(T)=\epsilon\left[\mathcal{P}_{k}\left(T, \mathbb{R}^{2}\right)\right]+J\left(b_{T}^{2} \mathcal{P}_{k-2}(T, \mathbb{R})\right)
$$

where $\epsilon$ is the infinitesmal strain operator (symmetrized gradient), $b_{T}$ is the cubic bubble function on $T$, and $J$ is Airy stress operator

$$
\tau=J q:=\left(\begin{array}{cc}
\partial^{2} q / \partial^{2} y & -\partial^{2} q / \partial x \partial y \\
-\partial^{2} q / \partial x \partial y & \partial^{2} q / \partial^{2} x
\end{array}\right) .
$$

Note that when $k=0, N_{k}(T)$ is simply the space of constant tensors.

Having given a unisolvent set of degrees of freedom for $V_{T}$ and $\Sigma_{T}$, our finite element space is assembled in the usual way. Let $\mathcal{T}_{h}$ be some triangulation of $\Omega$, i.e., a set of closed triangles with union $\bar{\Omega}$ and such that any two distinct non-disjoint elements of $\mathcal{T}_{h}$ meet in a common edge or vertex (henceforth we shall assume that $\Omega$ is a simply-connected polygonal region). The associated finite element element space $V_{h}$ is then the space of all piecewise linear vector fields with respect to this triangulation, not subject to any interelement continuity conditions. The space $\Sigma_{h}$ is the space of all matrix fields which belong piecewise to $\Sigma_{T}$, subject to the continuity conditions that the normal components are continuous across mesh edges and all components are continuous at mesh vertices. The first condition is necessary to ensure that $\Sigma_{h} \subset H(\operatorname{div}, \Omega, \mathbb{S})$, but the second condition is a further restriction not implied by the inclusion in $H(\operatorname{div}, \Omega, \mathbb{S})$. Table 1 gives the dimensions of these spaces for $k=1,2$, and Figure 1 shows the element diagrams.

TABLE 1. Local and global dimensions of the conforming finite element spaces. For the global dimensions, $v, e$, and $t$ are the number of vertices, edges, and triangles, respectively.

\begin{tabular}{ccccc}
\hline$k$ & $\operatorname{dim} \Sigma_{T}$ & $\operatorname{dim} V_{T}$ & $\operatorname{dim} \Sigma_{h}$ & $\operatorname{dim} V_{h}$ \\
\hline 1 & 24 & 6 & $3 v+4 e+3 t$ & $6 t$ \\
2 & 37 & 12 & $3 v+6 e+10 t$ & $12 t$ \\
\hline
\end{tabular}

For any value of $k$, we have $\operatorname{div} \Sigma_{h}=V_{h}$. It follows easily that there is a unique critical point of the Hellinger-Reissner function over $\Sigma_{h} \times V_{h}$, i.e., the mixed 

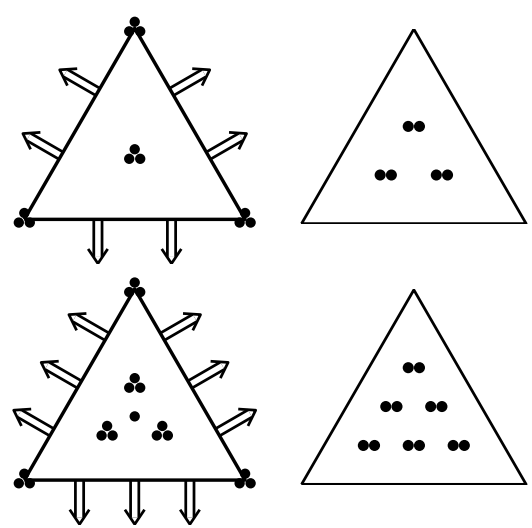

FiguRE 1. Element diagrams for the conforming elements in the cases $k=1$ and 2 .

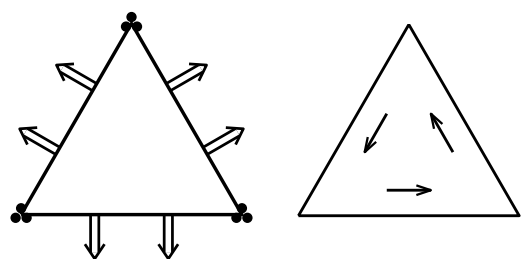

Figure 2. A simplified element pair.

method is well-defined. The following theorem, which is proven in [7], gives an error estimate.

Theorem 1. Let $(\sigma, u)$ be the unique critical point of the Hellinger-Reissner functional over $H(\operatorname{div} \Omega, \mathbb{S}) \times L^{2}\left(\Omega, \mathbb{R}^{2}\right)$ and let $\left(\sigma_{h}, u_{h}\right)$ be unique critical point over $\Sigma_{h} \times V_{h}$, where $\Sigma_{h}$ and $V_{h}$ are the spaces defined above for some integer $k \geq 1$. Then

$$
\begin{gathered}
\left\|\sigma-\sigma_{h}\right\|_{0} \leq c h^{m}\|\sigma\|_{m}, \quad 1 \leq m \leq k+2, \\
\left\|\operatorname{div} \sigma-\operatorname{div} \sigma_{h}\right\|_{0} \leq c h^{m}\|\operatorname{div} \sigma\|_{m}, \quad 0 \leq m \leq k+1, \\
\left\|u-u_{h}\right\|_{0} \leq c h^{m}\|u\|_{m+1}, \quad 1 \leq m \leq k+1 .
\end{gathered}
$$

There is a variant of the lowest degree $(k=1)$ element involving fewer degrees of freedom. For this we take $V_{T}$ to be the space of infinitesmal rigid motions on $T$, i.e., the span of the constant vectorfields and the linear vectorfield $\left(-x_{2}, x_{1}\right)$. Then we choose

$$
\Sigma_{T}=\left\{\tau \in \mathcal{P}_{3}(T, \mathbb{S}) \mid \operatorname{div} \tau \in V_{T}\right\} .
$$

We then have $\operatorname{dim} \Sigma_{T}=21, \operatorname{dim} V_{T}=3, \operatorname{dim} \Sigma_{h}=3 v+4 e, \operatorname{dim} V_{h}=3 t$. The element diagram is shown in Figure 2. For this element pair we have

$$
\begin{gathered}
\left\|\sigma-\sigma_{h}\right\|_{0} \leq c h^{m}\|\sigma\|_{m}, \quad 1 \leq m \leq 2, \\
\left\|\operatorname{div} \sigma-\operatorname{div} \sigma_{h}\right\|_{0} \leq c h^{m}\|\operatorname{div} \sigma\|_{m}, \quad 0 \leq m \leq 1, \\
\left\|u-u_{h}\right\|_{0} \leq c h\|u\|_{2} .
\end{gathered}
$$


All of our discretizations of $H(\operatorname{div} \Omega, \mathbb{S})$ involve vertex degrees of freedom. In this respect, they differ from the usual mixed elements for scalar elliptic problems, such as the Raviart-Thomas elements. As mentioned earlier, continuity at the vertices is not required for functions belonging to $H(\operatorname{div} \Omega, \mathbb{S})$. Moreover, it impedes the implementation of the elements using interelement Lagrange multipliers as in [3]. However, as we now explain, vertex nodes are unavoidable whenever continuous shape functions are used to construct an $H(\operatorname{div}, \Omega, \mathbb{S})$ finite element space. To see why, imagine building an $H(\operatorname{div}, \Omega, \mathbb{S})$ finite element space from spaces $\Sigma_{T}$ of continuous symmetric matrix fields, imposing interelement continuity only by means of quantities defined on the edges (and so shared by only two neighboring elements). This means that the degrees of freedom associated with each edge must determine the normal component on the edge. Now consider two edges of a triangle meeting at a common vertex $x$. If $n_{1}$ is the normal to the first edge and $n_{2}$ the normal to the second edge then the degrees of freedom on the first edge must determine $\tau n_{1}$ there and similarly the degrees of freedom on the second edge must determine $\tau n_{2}$ there. Since $\tau$ is continuous, we have in particular that the degrees of freedom on the first edge determine $\tau(x) n_{1} \cdot n_{2}$, and those on the second edge determine $\tau(x) n_{2} \cdot n_{1}$. But these quantities are equal since $\tau$ is symmetric. This is a contradiction, since the degrees of freedom on the two edges are necessarily independent. This argument indicates that it is at least necessary to take the quantity $\tau(x) n_{1} \cdot n_{2}$ as a degree of freedom associated to the vertex node $x$. But the node $x$ is shared by other triangles which will have other values for the edge normal vectors $n_{i}$. For this reason, except if we restrict to very special triangulations, we are forced to take all three components of $\tau(x)$ as degrees of freedom associated to the node $x$. Note that the composite $H(\operatorname{div}, \Omega, \mathbb{S})$ finite elements of $[\mathbf{1 5}]$ and [5] avoid the necessity of vertex degrees of freedom because they use discontinuous shape functions.

\section{Nonconforming elements}

In order to obtain simpler elements with fewer degrees of freedom, and, in particular, to avoid vertex degrees of freedom, in $[\mathbf{8}]$ we developed some low order nonconforming mixed finite element methods for elasticity.

The first element may be considered a nonconforming version of the $k=1$ conforming element. For $V_{T}$ we again take $\mathcal{P}_{1}\left(T, \mathbb{R}^{2}\right)$. For the stress shape functions we take

$$
\Sigma_{T}=\left\{\tau \in \mathcal{P}_{2}(T, \mathbb{S}) \mid n \cdot \tau n \in \mathcal{P}_{1}(e, \mathbb{R}), \quad \text { for each edge } e \text { of } T\right\} .
$$

The space $\Sigma_{T}$ has dimension 15, and a unisolvent set of degrees of freedom are

- the values of the moments of degree 0 and 1 of the two normal components of $\tau$ on each edge $e$ of $T$ (12 degrees of freedom)

- the value of the three components of the moment of degree 0 of $\tau$ on $T$ (3 degrees of freedom)

Otherwise stated, we determine $\tau \in \Sigma_{T}$ by giving the values of $\int_{e}(\tau n) d s$ and $\int_{e}(\tau n) s d s$ for all edges, and the value of $\int_{T} \tau d x$. (Here $s$ is a parameter giving the distance to one of the end points of $e$.) See the first pair of element diagrams in Figure 3. 

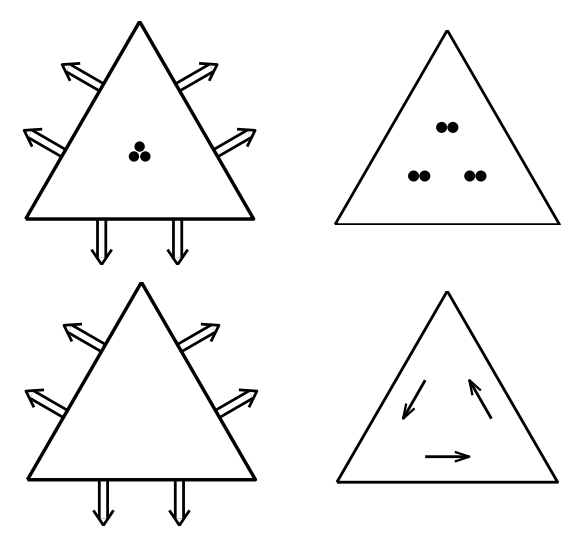

FiguRe 3. Two nonconforming mixed finite element pairs.

Note that the degrees of freedom associated to an edge do not determine $\tau n$ on that edge. Specifically, since the component $n \cdot \tau n$ is linear on the edge, it is entirely determined by the degrees of freedom associated the edge, while only the two lowest order moments of the quadratic $t \cdot \tau n$ are determined $(t$ is a unit vector tangential to $e$ ). Therefore, $\tau n$ may not vary continuously across element boundaries, and therefore $\Sigma_{h}$ is a nonconforming approximation of $H(\operatorname{div}, \Omega, \mathbb{S})$. Thus of course this means that the method introduces a consistency error. As show in [8], this consistency error can be bounded, and the method is first order convergent in the sense that

$$
\begin{gathered}
\left\|\sigma-\sigma_{h}\right\|_{0} \leq \operatorname{ch}\|u\|_{2}, \\
\left\|\operatorname{div} \sigma-\operatorname{div} \sigma_{h}\right\|_{0} \leq \operatorname{ch}^{m}\|\operatorname{div} \sigma\|_{m}, \quad 0 \leq m \leq 2, \\
\left\|u-u_{h}\right\|_{0} \leq \operatorname{ch}\|u\|_{2} .
\end{gathered}
$$

See $[8]$ for the proof.

Just as the conforming element shown on the left of Figure 1 can be simplified to element of Figure 2, the nonconforming element shown on the left of Figure 3 can be simplified to the element shown on the right of Figure 3. The displacement space consists of piecewise rigid motions, and the stress space is reduced by adding the restriction that the divergence be a rigid motion on each triangle. The dimension of the resulting space is 12 and the first two moments of the normal traction on each edge form a unisolvent set of degrees of freedom. For this element the $L^{2}$ error estimates for $\sigma$ and $u$ are the same as in (3.1), (3.3), but the error estimate (3.2) for the divergence holds only for $m=0,1$.

\section{Discrete differential complexes}

Important aspects of the structure of the plane elasticity system are summarized by the following differential complex:

$0 \longrightarrow \mathcal{P}_{1}(\Omega) \stackrel{\subset}{\longrightarrow} C^{\infty}(\Omega) \stackrel{J}{\longrightarrow} C^{\infty}(\Omega, \mathbb{S}) \stackrel{\text { div }}{\longrightarrow} C^{\infty}\left(\Omega, \mathbb{R}^{2}\right) \longrightarrow 0$.

Since we have assumed that $\Omega$ is simply-connected, this sequence is exact (i.e., the range of each map is the kernel of the following one). Thus this sequence encodes 
the fact that every smooth vectorfield is the divergence of a smooth symmetric matrix field, that the divergence-free symmetrix matrix fields are precisely those that can be written as the Airy stress field associated to some scalar potential, and that the only potentials for which the corresponding Airy stress vanishes are the linears polynomials. We have stated these results in terms of smooth functions, but analogous results hold with less smoothness. For example, the sequence

$0 \longrightarrow \mathcal{P}_{1}(\Omega) \stackrel{\subset}{\longrightarrow} H^{2}(\Omega) \stackrel{J}{\longrightarrow} H(\operatorname{div}, \Omega, \mathbb{S}) \stackrel{\operatorname{div}}{\longrightarrow} L^{2}\left(\Omega, \mathbb{R}^{2}\right) \longrightarrow 0$

is also exact. The well-posedness of the continuous problem-i.e., that for every $f \in L^{2}\left(\Omega, \mathbb{R}^{2}\right)$ there exists a unique $(\sigma, u) \in H(\operatorname{div}, \Omega, \mathbb{S}) \times L^{2}\left(\Omega, \mathbb{R}^{2}\right)$ there exists a unique critical point of (1.1), follows from this.

For each of the pairs of finite element spaces introduced above, $\left(\Sigma_{h}, V_{h}\right)$, we have $\operatorname{div} \Sigma_{h}=V_{h}$, i.e., the short sequence

$$
\Sigma_{h} \stackrel{\operatorname{div}}{\longrightarrow} V_{h} \longrightarrow 0
$$

is exact. (Note that, for the nonconforming methods, the operator div here must be understood as the piecewise defined divergence. We will continue with this abuse of notation below.) Moreover, if we define projections $P_{h}: C^{\infty}\left(\Omega, \mathbb{R}^{2}\right) \rightarrow V_{h}$ and $\Pi_{h}: C^{\infty}(\Omega, \mathbb{S}) \rightarrow \Sigma_{h}$ using the degrees of freedom which determined the finite element spaces, it can be shown that the following diagram commutes:

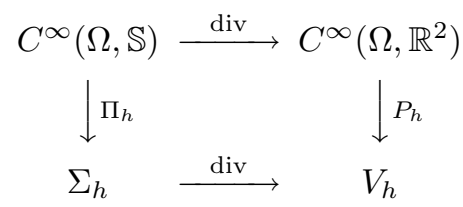

The stability of the mixed method follows from the exactness of (4.3), the commutativity of (4.4), and the well-posedness of the continuous problem.

It is natural to complete the sequence (4.3) to a resolution analogous to (4.1). For this purpose we define $Q_{h}=\left\{q \in H^{2}(\Omega) \mid J q \in \Sigma_{h}\right\}$. The $Q_{h}$ is a finite element approximation of $H^{2}(\Omega)$, conforming (i.e., a subspace of $H^{2}$ ) when $\Sigma_{h}$ is a conforming approximation of $H(\operatorname{div}, \Omega, \mathbb{S})$, and nonconforming when $\Sigma_{h}$ is nonconforming. Moreover, there is a natural construction of an interpolation operator $I_{h}: C^{\infty}(\Omega) \rightarrow Q_{h}$ so that following diagram with exact rows is commutative:

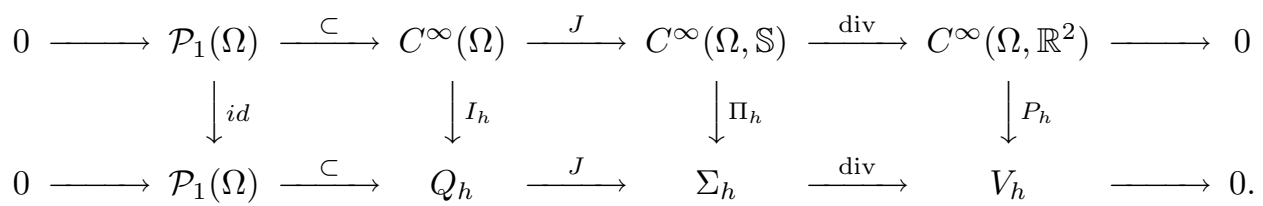

For a description of this construction, see [7]. As discussed there, under quite general conditions, the existence of a stable pair of spaces $\left(\Sigma_{h}, V_{h}\right)$ approximating $H(\operatorname{div}, \Omega, \mathbb{S})$ and $L^{2}\left(\Omega, \mathbb{R}^{2}\right)$, implies the existence of a finite element approximation $Q_{h}$ of $H^{2}(\Omega)$ related to $\Sigma_{h}$ and $V_{h}$ through the diagram above. This represents a substantial obstruction to the construction of stable mixed elements, and in part accounts for their slow development.

In particular, for the $k=1$ conforming elements show on the left of Figure 1 and for its reduced version shown in Figure 2, the corresponding conforming approximation $Q_{h}$ of $H^{2}(\Omega)$ is the well-known Hermite quintic or Argyris finite element $[\mathbf{2}]$; cf., also $[\mathbf{1 2}, \S 9]$. The local finite element space $Q(T)=\mathcal{P}_{5}(T, \mathbb{R})$ and the 

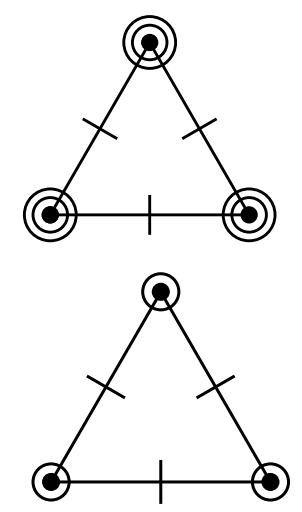
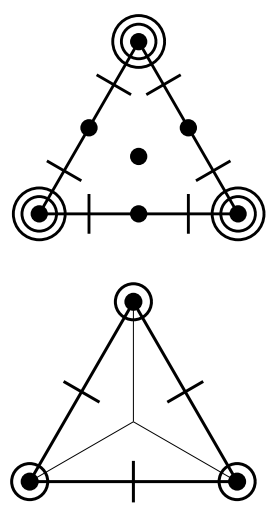

FiguRE 4. Element diagrams for $Q_{h}$ in the case of (a) our conforming elements for $k=1$ and (b) $k=2$, (c) our nonconforming elements, (d) the elements of Watwood-Hartz/Johnson-Mercier.

degrees of freedom are the values of the function and its partial derivatives of order 1 and 2 at the vertices and the averages of the normal derivatives on the edges; cf. Figure 4a. Thus the assembled space $Q_{h}$ consists of all $C^{1}$ piecewise quintics which are $C^{2}$ at the vertices.

Similarly, for the $k=2$ conforming elements, the corresponding conforming approximation $Q_{h}$ of $H^{2}(\Omega)$ is the Hermite sextic element, with $Q(T)=\mathcal{P}_{6}(T, \mathbb{R})$ and degrees of freedom as suggested by Figure $4 \mathrm{~b}$. The assembled space in this case consists of all $C^{1}$ piecewise sextics which are $C^{2}$ at the vertices.

For the nonconforming elements show in Figure 2, the space $Q_{h}$ turns out to be a nonconforming approximation of $H^{2}$ recently introduced by Nilsen, Tai, and Winther [17]. The local space $Q(T)$ consists of all quartic polynomials which reduce to cubic on the edges, and the degrees of freedom are the values of $q$ and the two first order partial derivatives of $q$ at each vertex, and the values of the moment of degree 0 of the normal derivative of $q$ on each edge $e$, as suggested in Figure 4b.

As mentioned, previous stable mixed element pairs for plane elasticity used composite elements. The first and simplest of these is the element of Watwood and Hartz [23], shown to be stable by Johnson and Mercier [15]. For this element $Q_{h}$ is the well-known Clough-Tocher conforming composite approximation of $H^{2}$, for which the element diagram is given in Figure 4c. For the higher order composite mixed elements introduced by Arnold, Douglas, and Gupta [5], the related $H^{2}$ finite element spaces are given by the higher order composite elements of Douglas, Dupont, Percell, and Scott [13]. Finally, we remark that the degrees of freedom for the nonconforming Nilsen-Tai-Winther element and the composite Clough-Tocher element are identical. It is then not suprising that the same is true for the nonconforming approximation of $H(\operatorname{div}, \Omega, \mathbb{S})$ shown on the left of Figure 3 and the composite element of Watwood-Hartz/Johnson-Mercier. So the corresponding finite element methods are closely related. Although the shape functions are very different, there is a one-to-one correspondence between the local bases. 


\section{References}

1. Mohamed Amara and Jean-Marie Thomas, Equilibrium finite elements for the linear elastic problem, Numer. Math. 33 (1979), no. 4, 367-383.

2. John H. Argyris, Isaac Fried, and Dieter W. Scharpf, The TUBA family of plate elements for the matrix displacement method, Aero. J. Roy. Aero. Soc. 72 (1968), 514-517.

3. Douglas N. Arnold and Franco Brezzi Mixed and nonconforming finite element methods: implementation, postprocessing and error estimates, Math. Modelling and Numer. Anal. 19 (1985), 7-32.

4. Douglas N. Arnold, Franco Brezzi, and Jim Douglas, Jr., PEERS: a new mixed finite element for plane elasticity, Japan J. Appl. Math. 1 (1984), no. 2, 347-367.

5. Douglas N. Arnold, Jim Douglas, Jr., and Chaitan P. Gupta, A family of higher order mixed finite element methods for plane elasticity, Numer. Math. 45 (1984), no. 1, 1-22.

6. Douglas N. Arnold and Richard S. Falk, A new mixed formulation for elasticity, Numer. Math. 53 (1988), no. 1-2, 13-30.

7. Douglas N. Arnold and Ragnar Winther, Mixed finite elements for elasticity, submitted to Numer. Math. 2001.

8. Douglas N. Arnold and Ragnar Winther, Nonconforming mixed elements for elasticity, submitted to Mathematical Models and Methods in the Applied Sciences (M3AS) 2002.

9. Franco Brezzi, On the existence, uniqueness and approximation of saddle-point problems arising from Lagrangian multipliers, Rev. Française Automat. Informat. Recherche Opérationnelle Sér. Rouge 8 (1974), no. R-2, 129-151.

10. Franco Brezzi, Jim Douglas, Jr., and L. Donatella Marini, Two families of mixed finite elements for second order elliptic problems, Numer. Math. 47 (1985), 217-235.

11. Franco Brezzi and Michel Fortin, Mixed and Hybrid Finite Element Methods, Springer-Verlag, New York, 1991.

12. Philippe G. Ciarlet, The finite element method for elliptic problems, North-Holland, Amsterdam, 1978.

13. Jim Douglas, Jr., Todd Dupont, Peter Percell, and Ridgway Scott, A family of $C^{1}$ finite elements with optimal approximation properties for various Galerkin methods for 2nd and 4 th order problems, RAIRO Anal. Numér. 13 (1979), no. 3, 227-255.

14. Baudoiun M. Fraejis de Veubeke, Displacement and equilibrium models in the finite element method, Stress analysis, (O.C Zienkiewics and G.S. Holister, eds.), Wiley, New York, 1965, pp. $145-197$.

15. Claes Johnson and Bertrand Mercier, Some equilibrium finite element methods for twodimensional elasticity problems, Numer. Math. 30 (1978), no. 1, 103-116.

16. Alain L. Mignot and Claude Surry, A mixed finite element family in plane elasticity, Appl. Math. Modelling 5 (1981), 259-262.

17. Trygve K. Nilsen, Xue-Cheng Tai, and Ragnar Winther, A robust nonconforming $H^{2}-$ element, Math. Comp. 70 (2001), 489-505.

18. Pierre-Arnaud Raviart and Jean-Marie Thomas, A mixed finite element method for 2nd order elliptic problem, in: Mathematical Aspects of the Finite Element Method, Lecture Notes in Mathematics 606, Springer-Verlag, 19977, 292-315.

19. Erwin Stein and Raimund Rolfes, Mechanical conditions for stability and optimal convergence of mixed finite elements for linear plane elasticity, Comput. Methods Appl. Mech. Engrg. 84 (1990), no. 1, 77-95.

20. Rolf Stenberg, On the construction of optimal mixed finite element methods for the linear elasticity problem, Numer. Math. 48 (1986), no. 4, 447-462.

21. Rolf Stenberg, A family of mixed finite elements for the elasticity problem, Numer. Math. $\mathbf{5 3}$ (1988), no. 5, 513-538.

22. Rolf Stenberg, Two low-order mixed methods for the elasticity problem, The mathematics of finite elements and applications, VI (Uxbridge, 1987), Academic Press, London, 1988, pp. 271280.

23. Vernon B. Watwood Jr. and B. J. Hartz, An equilibrium stress field model for finite element solution of two-dimensional elastostatic problems, Internat. Jour. Solids and Structures 4 (1968), 857-873. 
Institute for Mathematics and its Applications, University of Minnesota, MinNEAPOLIS, MiNnESOTA 55455

E-mail address: arnold@ima.umn.edu

Department of Informatics, University of Oslo, Oslo, Norway

E-mail address: rwinther@ifi.uio.no 